Author affiliations and support information (if applicable) appear at the end of this article.

Published at jco.org on August 29, 2018 Corresponding author: David J. Einstein, MD, 330 Brookline Ave, Boston, MA e-mail: deinstei@bidmc.harvard.edu.

(C) 2018 by American Society of Clinical Oncology

$0732-183 X / 18 / 3629 w-2969 w / \$ 20.00$
DOI: https://doi.org/10.1200/JCO 18.00205

\title{
Compassion and Compassionate Use
}

David J. Einstein

The e-mail came down to this: "This patient is running out of options, and he and his family are starting to think 'outside the box."' Two months prior, the sender was my cofellow. Now, after a blissful vacation month entirely unplugged from oncology, he and I were junior attending physicians, launched into the dual frontlines of inpatient and outpatient oncology. He was staffing the consult service, I, the inpatient oncology service, both of us simultaneously trying to keep our back-burner outpatient clinics and research projects from boiling over. He was letting me know about my newest admission, a patient with advanced choriocarcinoma, which was ostensibly within my area of expertise of genitourinary cancers.

During years of residency training, we had learned to eyeball patients and differentiate sick from not sick-or really, those who might be imminently dying from those who were okay for now. As oncology fellows, we recognized a new group, the patients who seemed to be imminently dying on paper but in person looked amazingly normal. The patient referred to in the e-mail was such a patient, an energetic engineer in his fifties, with a more-than-slight Boston accent despite his years in California and Texas, and plenty of Red Sox gear to match. On paper, he was in dire shape, with treatment-resistant advanced cancer and increasing toxicities of treatment.

The patient and his family had taken on his disease with the battle mentality that works for some and makes others cringe. After multiple rounds of chemotherapy, resections, and radiation treatments failed to cure his disease, he moved on to the high-dose chemotherapy with autologous stem cell rescue that is the standard of care in this setting. If some chemotherapy is good, more is better. However, he eventually exhausted his supply of stem cells, still without having achieved remission. Time to think outside the box. Turning to his identical twin brother as a source of new stem cells, the patient proceeded to a last-ditch effort at high-dose chemotherapy, this time with syngeneic transplantation. It worked-for approximately 3 months. Despite the most intensive efforts, his disease kept bouncing back. At this point, it was clear that chemotherapy would only transiently suppress his cancer but not cure it. Meanwhile, his treatments left him with hearing loss severe enough to require hearing aids, poor kidney function, foot numbness, and bone marrow that even on imaging looked exhausted, plus prolonged cytopenias to prove it.

What could be done? This patient and his family had crossed the country in search of an outside-the-box treatment that might offer him the benefit that standard treatments had not. We are used to patients looking online for new diets and complementary medicines; we understand that this is a way to seek an active part in their care when they otherwise feel like passive recipients. The patient and his family had gone further, identifying a clinical trial available at our hospital that they desperately wanted to join. But the trial was in a different tumor type, and moreover, he would not be a candidate for any kind of trial, given his accumulated treatment-related toxicities. Undaunted, his sister found an online poster from our peer institution across the street describing a novel compound used to treat a patient with his disease, with a resulting (and seemingly miraculous) remission. Again, we are accustomed to the patient who brings in the latest online research to an appointment. Sometimes, it is not scientifically valid, sometimes it is valid but not applicable to the situation, and occasionally, it is both valid and applicable and actually does result in a treatment change. As I scrutinized the information the patient's sister provided, it actually seemed both possibly valid and applicable. I could find no obvious toxicity that precluded use of this drug. And yet, it was early in testing, far from full approval. "What about compassionate use?" the family asked.

"Compassionate use" was a term I had only heard spoken of dismissively. We oncologists prided ourselves on always being compassionate, whether we were administering therapies or stopping them. Treating outside of standard of care without the structure of a research study is dangerous territory. But this concept has a popular appeal: who wouldn't want to try anything 
that could help in a desperate situation? Recently, proponents of so-called right-to-try legislation claimed that Food and Drug Administration (FDA) regulation was interfering with patients' access to experimental therapies outside of trials; the FDA replied that they approved $99 \%$ of expanded-access requests. Indeed, compared with the regulatory hurdles I faced from my own institution, I discovered that the FDA was the smallest barrier of all.

As I learned, creating a single-patient Investigational New Drug application for compassionate use was the ultimate in personalized medicine- a clinical trial created for one person. In fact, it was a desperate hope thrown into the slow-moving and opaque gears of our clinical research regulation, only to be chewed into a million tracked-changes documents and flurries of e-mails. I was rapidly assembling a trial protocol to use highly experimental therapy in a patient whose disease was bound to cause suffocation or bleeding at any moment. It was a terrifying, anxiety-provoking situation for physician, patient, and family alike. I felt caught between, on the one side, an eager and aggressive patient who wanted to sign anything, cover any cost, just to have a shot at a miracle, and on the other side, a process purposefully built to be as cautious as possible. I caught myself erupting in frustration, unfairly lashing out at the secretary who e-mailed me with institutional review board (IRB) edits on an informed consent form that the patient would never read.

Just as we were getting ready to sign this consent form, the patient's disease was making itself more plainly visible in the form of a bleeding superficial metastasis. Now, another regulatory catastrophe: between the bleeding lesion and the need for radiation, he was ineligible for the "trial" because of the strictly worded inclusion criteria used in the original protocol. Time for yet another urgent amendment, pleading for the IRB not to hold me to the rules that I myself had provided.

When, after months of work, the patient received a single dose of the study treatment, we all felt like we had climbed Everest. All along the way, I tried to frame this as a victory in and of itself, hoping he would die feeling he had tried everything. But he, of course, wanted to live, through sheer will and perseverance. And, I'll admit, a small portion of me wanted to prove my doubts wrong, to be the junior attending daring enough to try something audacious and land a huge, unconventional win.

It was not to be. After many tense clinic appointments and late-night calls during my drive home, our last conversations unfortunately occurred in the intensive care unit. With the rush of the high-flow nasal cannula as background white noise, I told the patient that his disease was not even responding to the chemotherapy I had tried a week ago in an ultra-last-ditch effort to get him back to the study drug. I could not pull him back from the edge of that ultimate cliff. Although I had tried hard to have the appropriate discussions with the patient and his family on many occasions-trying to refocus their hopes on spending good time together, freedom from pain and breathlessness, and another day outside the infusion area and the oncology ward-I knew they felt I had failed them, that maybe pushing just a little harder would have made the difference. One morning, I awoke to an e-mail from the intensive care unit resident letting me know that the patient had died that night. Thankfully, we had agreed that aggressive interventions in his dying moments would be even less effective than our chemotherapies, and instead, he had received much more effective supportive care.

I let out a breath of simultaneous relief and regret. I knew this moment had been coming, of course. I had disagreed with my patient and his family about how to prepare for it, but my team and I had tried to make the patient and his family feel that every effort had been made on his behalf. I felt the failure not in his inevitable death, but in never arriving at a moment of shared celebration for our combined efforts, when we could say together, even in that intensive care unit room, "It was all worth it." And when I went to contact his wife and sister, I never heard from them again. I certainly did not fault them for not returning my calls or e-mails during the midst of intense grief. Yet, I never heard whether they felt that our compassionate use of a single dose of experimental treatment had helped or hurt. He went from being my allconsuming focus to a vanished spirit, and I had to wonder whether the family's silence gave me my answer.

The recent right-to-try legislation cleared the US Senate last year and the House of Representatives in March, along mostly partisan lines. Opponents, including representatives from the American Society of Clinical Oncology as well as the American Cancer Society, pointed out the value of FDA oversight and the existing pathway for access to experimental therapies outside of clinical trials. Regardless of the fact that this legislation does not affect the institutional barriers I encountered, I do wonder whether it will inspire a growing number of desperate patients to exercise their right to try.

How should oncologists respond? Except in the cases of a drug that seems to have particularly exciting preclinical justification or early clinical data, I suspect most will advise their patients with advanced cancer to stick to more trodden territory: to try accepted anticancer therapies when they are judged to be reasonable and to switch to supportive care alone when the risk seems to outweigh the benefit (individualized to patient preferences). "As we continue to chase progress in cancer," Wachter ${ }^{1}$ recently wrote, "let's be sure that we don't rob dying patients of a smaller, more subtle miracle: a death with dignity and grace, relatively free from pain and discomfort." But there will always be the patients like mine, unsatisfied to do nothing, as much as we try to reinforce that intensive supportive care is far from nothing. I recall the words of another oncologist who had seen the patient for a second opinion: "These patients [with refractory testicular cancer] die with the chemo going in." Chemotherapy or, perhaps increasingly, experimental drugs.

If we are to routinely offer such patients access to experimental drugs outside of clinical trials, then we will need to address several issues in advance. First, federal and local regulatory burdens will have to be substantially lower. We will need to simplify the process of writing a protocol, obtaining IRB approval, and amending the protocol as clinical circumstances evolve. (As a word of advice, oncologists embarking on such a process should be careful about recycling protocols written for standard trials; in particular, because these protocols are designed around a specific patient, the inclusion and exclusion criteria should be minimal.) Second, directors of clinical trial programs will have to decide whether their operations are capable of diverting resources from standard trials to these urgent and unfunded projects. Finally, and most importantly, individual clinicians will have to develop strong end-of-life communication skills to help frame a shared decisionmaking process properly, and they may even have to make 
especially difficult decisions about when to exercise a right to deny such requests. I hope that all of my patients will feel that I have treated them compassionately and with the tools best suited to their situation-whether or not this includes compassionate use of unproven therapies. And I hope that my patient did indeed feel that we left no stone unturned and that he felt some small satisfaction in this.
AUTHOR'S DISCLOSURES OF POTENTIAL CONFLICTS OF INTEREST Disclosures provided by the author are available with this article at jco.org.

\section{REFERENCE}

1. Wachter RM: The problem with miracle cancer cures. New York Times, April 19, 2018: SR5

\section{Affiliation}

David J. Einstein, Beth Israel Deaconess Medical Center, Boston, MA.

\section{Support}

Supported by Congressionally Directed Medical Research Programs (CDMRP) Prostate Cancer Research Program (PCRP) Physician Research Award \#W81XWH-17-1-0350.

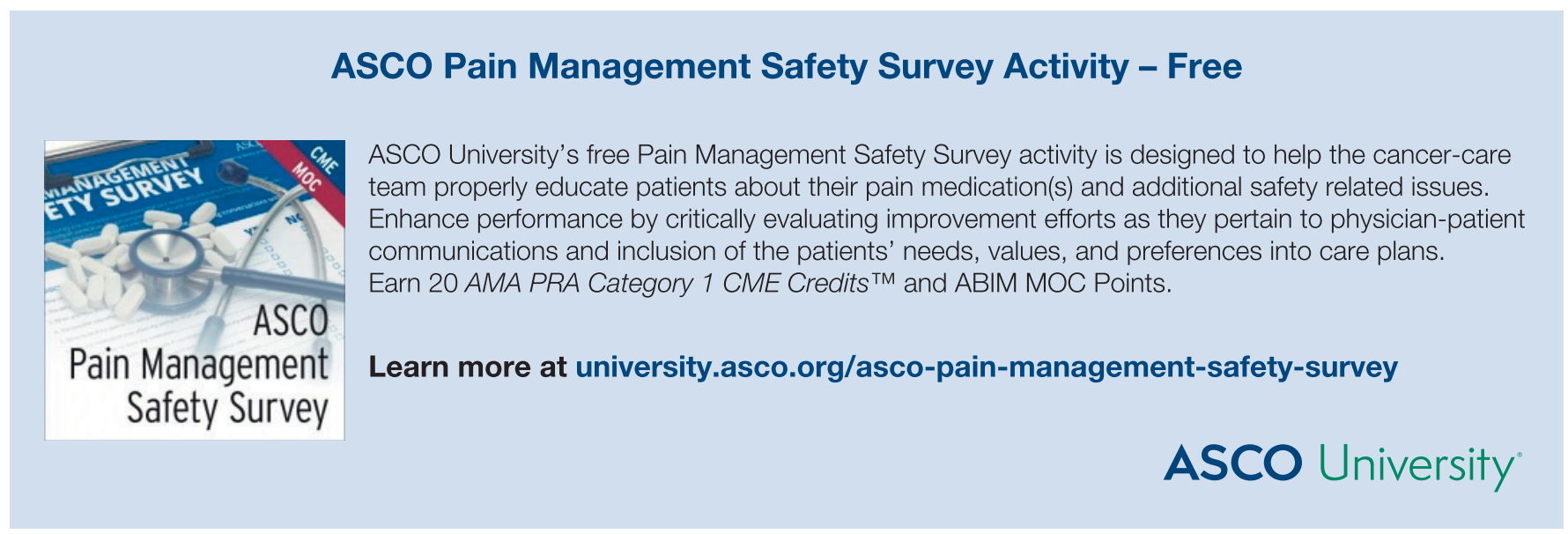




\section{AUTHOR'S DISCLOSURES OF POTENTIAL CONFLICTS OF INTEREST}

\section{Compassion and Compassionate Use}

The following represents disclosure information provided by authors of this manuscript. All relationships are considered compensated. Relationships are self-held unless noted. I = Immediate Family Member, Inst = My Institution. Relationships may not relate to the subject matter of this manuscript. For more information about ASCO's conflict of interest policy, please refer to www.asco.org/rwc or ascopubs.org/jco/site/ifc.

\section{David J. Einstein}

Research Funding: Trovagene (Inst), Bristol-Myers Squibb (Inst),

Oncbiomune (Inst) 